\title{
The Continuing Evolution of Precision Health in Type 2 Diabetes: Achievements and Challenges
}

Yuan Lin ${ }^{1}$ MD, PhD; Jennifer Wessel ${ }^{1,2,3} \mathrm{PhD}, \mathrm{MPH}$

${ }^{1}$ Department of Epidemiology, Richard M. Fairbanks School of Public Health, Indiana University, Indianapolis, IN, USA

${ }^{2}$ Department of Medicine, Indiana University School of Medicine, Indianapolis, IN, USA

${ }^{3}$ Diabetes Translational Research Center, Indiana University School of Medicine, Indianapolis, IN, USA

\section{Corresponding Author:}

Jennifer Wessel

410 W 10 th Street, Suite 3100

Indianapolis, IN

46202

wesselj@iu.edu

\section{Co-author email:}

linyy@iu.edu

Keywords Type 2 Diabetes; Precision Diabetes; Review; Genetics; Genomics; Heterogeneity

\begin{abstract}
Purpose of Review The purpose of this review was to summarize recent advances in the genomics of type 2 diabetes (T2D) and to highlight current initiatives to advance precision health.

Recent Findings Generation of multi-omic data to measure each of the 'biologic layers', developments in describing genomic function and annotation in T2D relevant tissue, along with the increasing recognition that T2D is a heterogeneous disease, and large-scale collaborations have all contributed to advancing our understanding of the molecular basis of T2D.

Summary Substantial advances have been made in understanding the molecular basis of T2D pathogenesis, such that precision health diabetes is increasingly becoming a reality. For precision diabetes to become routine in clinical and public health, additional large-scale multiomic initiatives are needed along with better assessment of our environment to delineate an individual's diabetes subtype for improved detection and management.
\end{abstract}

This is the author's manuscript of the article published in final edited form as: 


\section{Introduction}

Diabetes has become a global pandemic and the burden of this disorder is enormous. The estimated worldwide prevalence of diabetes among adults is predicted to be as high as 439 million (7.7\%) by 2030 , with type 2 diabetes (T2D), the predominant form of diabetes, accounting for at least $90 \%$ of cases [1]. As a complex disease, the importance of genetic contributions to T2D, and their interaction with pervasive environmental exposures, has long been accepted [2]. However, in nearly every case, the factors that affect disease causation are still largely unknown, and the mechanistic understanding we have of T2D is lacking [3]. Now it is increasingly being recognized that T2D is a highly heterogeneous syndrome. Individuals may share the same diagnostic label but have very heterogeneous presentations and clinical courses; resulting in very different responses to the suite of available lifestyle or therapeutic interventions [4]. These observations motivate research in the field of precision diabetes, which ideally will tailor T2D prevention and therapy to individual patients for enhanced tolerability and effectiveness.

\section{Genomics of T2D}

Our knowledge of the human genome has increased substantially since the completion of the Human Genome [5] and HapMap Projects [6]. This heralded advances in genotyping technology, and along with rapid improvements in computing technology, the implementation of genome-wide association studies (GWAS) for complex diseases has become a reality. The study of the genetics of T2D has been at the forefront of this genetic revolution. To date, over 400 loci have been robustly associated with T2D; and hundreds of loci have been identified as contributing to variation in T2D-related traits [7, 8].

Integration of genetic and genomic information, followed up with well-designed functional studies, provide increased resolution of the molecular mechanisms through which variants underlying association signals exert their effects on T2D pathogenesis. For instance, one study conducted a comprehensive fine-mapping of 39 established T2D loci in 27,206 cases and 57,574 controls of European ancestry. Through statistical fine-mapping (e.g. conditional, credible set and genomic enrichment analyses) 49 distinct association signals were identified. To interrogate the 39 non-coding variants, credible set results were overlapped with islet and liver specific functional and regulatory annotation sites, of which only 11 could be shown to be in transcriptionally active regions. To demonstrate how diabetes-specific genomic enrichment analyses can be used to highlight regulatory mechanisms the MTNR1B locus was chosen for functional validation. The MTNR1B rs10830963-G allele was shown to affect NEUROD1 binding ability in human islet cells [9]. The pursuit of causal variants at genetic loci is shedding light on the underlying mechanisms; however, to date, biological function has been determined for only a handful of loci, highlighting the challenge of going from association to function.

Collectively, genome-wide-significant and validated SNPs account for $10-15 \%$ of overall heritability of T2D susceptibility [10]. This "missing heritability" led to next generation sequencing of whole exomes and genomes to identify rare variants with presumably larger genetic effect sizes [11]. Initial results from these studies did not support the idea that rare variants have a major role in predisposition to T2D [12]; however as was observed with GWAS, increasing sample size, along with better imputation panels, has led to additional rare variants being identified, and their effect sizes actually range from modest to large (1.08-8.05) [7].

At the same time, in-depth genetic association studies in specific ancestries or populations have highlighted the heterogeneity of the genetic basis of T2D. Variants that are rare in the global population account for a small overall burden of diabetes, but are found to be common in 
specific ancestral populations and are associated with a greater increase in diabetes risk. For example, a variant at the HNF1A [13] locus has only been associated with T2D in Latino populations, or a TBC1D4 variant only confers T2D risk in the Inuit populations of Greenland [14], and a PAX4 risk allele that was only observed in individuals of East Asian ancestry [12].

The discovery of multiple variants associated with risk for T2D has resulted in the development of genetic risk scores (GRS), a widely used approach to assess the overall contribution of known genetic susceptibility loci to complex diseases. While the GRS is a simple and effective tool, early use of GRS did not improve the prediction of diabetes beyond traditional nongenetic risk factors, and therefore showed limited clinical validity [15]. Recently a genomewide polygenic score (GPS) has been created to identify individuals at clinically significant increased risk of common diseases with polygenic inheritance, including T2D [16]. Clinically significant risk was defined as threefold increased risk, equivalent to the risk observed in monogenic or familial inheritance of common diseases. The polygenic predictor for T2D identified $3.5 \%$ of the population with at least threefold increased risk and the top $1 \%$ had 3.30 fold increased risk compared to the remainder of the UK Biobank study participants. The most recent study aggregating genome-wide genotyping and imputed data ( $27 \mathrm{M}$ variants) from 32 European-descent GWAS (74,124 T2D cases, 824,006 controls) identified 403 T2D-risk signals, including both common and rare variants. Leveraging the combined set of GWAS data, a GPS was generated and further validated in the UK Biobank population. Individuals in the top $2.5 \%$ of the score distribution (T2D prevalence $=11.2 \%$ ) were at $>9$-fold increased risk compared to the bottom $2.5 \%($ T2D prevalence=1.2\%)[7]. This finding suggests the increasing value of polygenic risk prediction.

\section{Multi-omics: Beyond Genetic Variation}

Led by genomics, other high-throughput technologies have been applied more recently to search for new biomarkers of T2D beyond the individually static genome. A promising approach, metabolomics, has shown its value in the identification of circulating small molecules, such as branched-chain amino acids, acylcarnitines and aromatic amino acids, that can serve early detection and diagnosis of T2D [17-21]. Advances have also occurred in proteomics; the Human Proteome Organization is opening a new door to better understand T2D pathology through the application of proteomics to improve identification of persons at risk and improvement of current therapies [22]. At the same time, modulation of gene expression by epigenetic modifications and the action of microRNAs have been recognized as critical processes affecting T2D risk [23]. Moreover, convincing evidence suggests that compared to healthy controls, profiles of the gut microbiome in patients with T2D have compositionally changed [24]. These approaches are allowing investigators to explore complementary biological axes in greater breadth and depth. These efforts are promising but are still emerging in terms of developing a framework of accepted standards e.g. analytic methods, interpretation and reproducibility of results.

\section{The Precision Problem in Diabetes}

Precision health considers the unique genetic and environmental risks factors of an individual to prevent and treat disease. T2D and prediabetes represent multifactorial disease states on a continuum between normal and disrupted control of glucose metabolism. The pathophysiologies underlying T2D largely separate into features related to impairment of insulin secretion (i.e. pancreatic islet $\beta$-cell dysfunction) and features related to impairment in insulin sensitivity (i.e. tissue responses to insulin). The goal of delivering targeted care to individuals with T2D, and targeted prevention to individuals with prediabetes, is hampered by a lack of specificity and precision in these diagnoses (Figure 1). Individuals differ in the relative contributions of impaired 
$\beta$-cell function versus impaired insulin sensitivity, and simple disease state definitions do not reflect the balance between these components.

\section{T2D is a Heterogeneous Disease}

Historically, T2D has been seen as a homogeneous condition. As a result, therapeutic interventions (i.e. lifestyle or medications) have largely been applied to the population as though it is uniform in its pathology. There is increasing evidence, however, that there are a myriad of factors that differentiate what we currently term T2D, and in fact we now know that what has historically been viewed as a single disease may best be thought of as deriving from distinct mechanisms that result in similar clinical manifestations $[2,25,26]$. There are multiple lines of evidence that point towards T2D as being heterogeneous. Epidemiologic studies describe disparate distributions of diabetes across groups (e.g. ethnicity, weight trajectories, lean vs. obese) [26-28]. Studies comparing the prevalence of impaired fasting glucose (IFG) and impaired glucose tolerance (IGT) have consistently reported that these define two distinct and only partially overlapping populations [29-32]. This suggests that IFG and IGT represent intermediate states of glucose intolerance resulting from different pathophysiologic etiologies that result in the same diagnosis. This is further supported by animal models of diverging susceptibilities resulting in changes in expression of metabolic phenotypes or T2D development [33-36]. Functional follow-up studies from GWAS results have shown that a range of biological processes (e.g. glucagon-like peptide 1-stimulated insulin secretion, insulin exocytosis to posttranscriptional processing of insulin) are involved in the pathogenesis of T2D [37-40].

Additionally, the impact of T2D susceptibility variants on quantitative glycemic traits reveals mechanistic heterogeneity in T2D [41]. Lastly, differential response to lifestyle [42-45] and drug [46] therapies have been established.

\section{Characterizing the heterogeneity of T2D}

Recently, "integrative" (or systems) approaches have been used to successfully identify disease subtypes that correlate with meaningful clinical outcomes, most notably with cancer. For example, integrating genomics data to define subtypes of luminal breast cancer identified a clinical subset known to be non-responsive to available treatments [47]. Specific molecular aberrations were found that were unique to the clinical subset, which pointed to promising drug targets.

In an effort to define the heterogeneity observed in T2D, four recent studies have used a number of methods, measures and populations to identify T2D subtypes [48-51]. First, a 'pure' electronic medical record (EMR) approach has been applied in a data-agnostic manner to identify clinical features of individuals with T2D that self-aggregate and identify different clinical prognoses [48]; these clusters were subsequently associated with genetic signatures based on GWAS-derived T2D associated gene variants. The second approach integrated molecular multiomic genome-wide gene expression and methylation data to define molecular subtypes of T2D; two subtypes were identified and associated with different clinical characteristics [49]. Third, a targeted set of clinical and biochemical data collected at the time of diagnosis of T2D was used to identify diabetes subtypes [50]. This method produced clinically meaningful diagnostic clusters, in particular identifying groups with distinct clinical prognoses. A subsequent GWASbased gene variant association analysis identified some genetic signatures that related to these clinical diagnostic clusters. A fourth study applied a purely genetic approach since germline genetic markers have the advantage of being static; i.e. do not change with disease progression or treatment. T2D-genetic loci were first clustered into groups representing different mechanistic pathways, related to insulin secretion, anthropomorphic, and insulin response traits. These clusters of genetic loci were then used to deconstruct T2D heterogeneity by associating these 
clusters with T2D clinical outcomes; i.e. coronary artery disease, stroke and blood pressure [51]. These approaches represent advances in our ability to sub-classify individuals with T2D.

Indeed the 'palette' model for diabetes was recently proposed [4]. The model centers on a molecular taxonomy and shifts the focus of understanding the disease architecture to the major pathophysiological processes of an individual (e.g. obesity, fat distribution, islet development and function, and insulin sensitivity) that contribute to diabetes risk and progression. Individuals with diabetes will have multiple parallel defects that affect several of these processes, and each of these processes is under multifactorial (genetic and nongenetic) control. This review proposed that research efforts should, at least initially, be targeted towards identifying and characterizing individuals whose adverse metabolic trajectory is dominated by perturbations in a restricted set of processes.

\section{Opportunities to Advance Precision Health of T2D}

\section{'Big Data' Approaches}

An abundance of "omic" data and deeper annotations of the human genome are available through consortia and public resources; and new methods have been developed for integrating and analyzing "omic" data. Technological advances have created multiple genome-scale molecular platforms of genetic variation, sequence, gene expression, methylation, miRNA, proteomic and metabolomic data that is increasingly easy to measure in population studies. Elucidating the molecular drivers of T2D requires a strategy that can integrate multiple forms of molecular or "omic" data on large sample sizes that are most likely mechanistically heterogeneous. An "integrative genomics" approach has been proposed as a method that could change our understanding and treatment of diabetes [52, 53]. A number of integrative approaches have been developed $[54,55]$ and effectively applied to identify cancer subtypes $[47,56]$ demonstrating significant improvement over histologic subtyping [57] and novel mechanisms for developing drug targets [55]. To better characterize subtypes of T2D, integration of multi-platform "omic" data can be leveraged against rich physiologic and clinical data on multiple study populations that is available to investigators (e.g. dbGaP or consortia). Large-scale projects have improved annotation of functional elements of the human genome [58], gene expression and regulation across multiple tissues [59-61]. Relevant to T2D is understanding the regulation of genes in islet cells [62-64]. Investigating each biologic 'layer' (e.g. DNA, transcription, protein) can provide a clearer picture of the pathophysiologic processes contributing to overt T2D. Technological advances have created genome-scale data of these biologic layers.

\section{Large-scale Collaborations}

The T2D Knowledge Portal (www.type2diabetesgenetics.org/) is a worldwide scientific consortium of collaborators from academia, industry, government, and non-profit organizations. The portal provides access to comprehensive human genetic information (results from GWAS, exome chip, exome sequencing, and whole-genome sequencing (WGS) studies) associated with T2D, glycemic measures and other related traits. Functional and epigenomic data, and clinical outcome studies on T2D and its macro- and microvascular complications are being integrated into the database with the goal of identifying and validating genetic determinants of the onset of T2D, disease severity, or disease progression, creating analytic tools to benefit customized analyses, and further assist in the selection of new diabetes targets for drug design [65].

Global research consortia and collaborative networks are also rapidly growing in the field of precision health in diabetes (adapted from [66]). In 2005, a national and international health 
resource, the UK Biobank (https://www.ukbiobank.ac.uk/about-biobank-uk/) was established with the aims of improving the prevention, diagnosis and treatment of a wide range of serious illnesses - including diabetes. UK Biobank leverages medical history questionnaire data and electronic health records on 500,000 volunteers, which allows for follow up of clinical outcomes. In addition, blood samples have been collected for detailed measures, such as, genotyping and biomarkers (e.g. hormones and cholesterol). Data collected by the UK Biobank are made available for approved researchers in the UK and overseas to undertake health research for public benefit. Another European partnership program for health, the Innovative Medicines Initiative (IMI), launched 10 years ago with the focus on the major diseases affecting European citizens. IMI is investigating multiple biomarkers to identify patients who can be stratified by treatment efficacy. Since 2010, more countries have become involved in precision health of diabetes, with the implementation of national precision health programs (including the U.S. Precision Medicine Initiative, Australian Precision Medicine Initiative, Chinese Precision Medicine Initiative, Nordic Precision Medicine Initiative and Saudi Human Genome Project).

As the overarching goal of precision health is to personalize approaches toward improving health and treating disease, implementation of clinical trials with innovative evidence generated from research is a crucial intermediate link. There is a great need for strengthening the national capacity to implement cost-effective large-scale clinical trials. The NIH Health Care Systems Research Collaboratory (http://rethinkingclinicaltrials.org/) is making such efforts by improving the way clinical trials are conducted. The Collaboratory creates a new infrastructure for collaborative research with healthcare systems and supports the design and rapid execution of pragmatic clinical trials. The ultimate goal is to ensure that healthcare providers and patients can make decisions based on the best available clinical evidence.

\section{New efforts in precision diabetes}

President Obama announced the launch of a new precision health initiative to "bring us closer to curing diseases like .... diabetes" in 2015 [67]. Since then, a number of large-scale projects have emerged from the National Institutes of Health and others. For example, a diabetes precision health effort, local to the authors, is the Indiana University Grand Challenge Precision Health Initiative (PHI) launched in 2016 [68]. \$120 million is being invested to transform biomedical research and public health by building research and educational capacities, to lead research in diabetes precision health. The Diabetes PHI began in 2018 with the aim of characterizing individuals according to their precision diabetes subtype, which will inform risk assessment and guide the clinician to individualized approaches to prevention and therapy.

The National Heart, Lung, and Blood Institute (NHLBI) Trans-Omics for Precision Medicine (TOPMed) project began in 2014 with the goal of improving the understanding of heart, lung blood and sleep disorders, and to advance precision medicine [69]. The innovation of the project is the collection and generation of WGS and multi-omic data, e.g. gene expression, metabolome and proteome. The generation of these data is ongoing and currently over 150,000 individuals will have their genomes sequenced and many will have multi-omic data measured that will be integrated with extensive phenotyping data already generated on the NHLBI prospective and case-control studies. Analyses of T2D and glycemic traits using the recent release of WGS data (n up to 47,000) are nearing completion and investigators are expected to publish their initial discovery papers in 2019. Data will also be available to eligible investigators who can access dbGaP.

The enormous volume of genetic and multi-omics data that are being derived from diverse population studies, large multi-study collaborations and big-data efforts have become the next stage in contemporary genetic epidemiology. The TOPMed program has created a big-data environment on a cloud-based platform, Analysis Commons, which combines multi-omic data, 
phenotypic data as well as clinical information across studies [70]. This setting relies on a new team-science model, which incorporates collaborative resources into a single shared analytic platform with computationally efficient analysis and methodological developments. The Analysis Commons is a blueprint of how to address the practical issues of both large-scale computing and collaboration that many studies are now facing.

\section{Applying Precision to Treatment and Prevention of T2D}

Precision in diabetes prevention today consists of individualizing the approach to lifestyle intervention (Figure 1). Lifestyle modification has been demonstrated to be effective at reducing T2D risk [71-73]. More so, several studies observed that lifestyle interventions can attenuate the risk of T2D even among individuals with a higher genetic susceptibility, suggesting the effects are independent of genetic burden $[45,74]$. In light of the increasing prevalence of obesity and T2D along with the difficulty in changing and sustaining healthy lifestyles, it might be helpful to understand whether genetic information or other precision measures can be used to predict the individual-level benefits of lifestyle modifications [75]. Moreover, it is of greater value to determine which specific behaviors an individual could modify that will deliver the biggest return and sustainability of healthy behaviors. One example of such an effort related to diabetes has been published recently [76]. Incorporating data on dietary habits, blood parameters, physical activity, anthropomorphic, and gut microbiota, the postprandial glycemic response can be predicted by a machine-learning algorithm. A clinical trial applied this new approach and demonstrated improvement in glycemic outcomes. Mutie et al. systematically reviewed the literature on lifestyle interventions and diabetes prevention, discussing the limitations of existing approaches and describing the ideas behind precision lifestyle interventions, for instance, differential response to lifestyle interventions based on unique genetic traits [77]. Now that the concept of lifestyle precision health is becoming increasingly accepted, the National Institutes of Health's Common Fund Initiative on the Molecular Transducers of Physical Activity in Humans is currently underway. This effort is designed to determine the molecular mechanisms that define how physical activity benefits human health. Over the course of the program, up to 3,000 participants across the U.S. from diverse cultural and ethnic backgrounds with varying degrees of physical activity will participate in the study to generate a molecular map of changes that occur with physical activity. This knowledge will, in the future, allow researchers and clinicians to develop individually targeted exercise recommendations as well as develop therapeutics for those unable to exercise.

The leading-edge application of precision health in diabetes treatment consists of evaluating whether genetic information or other precision measures can be used to predict treatment response (Figure 1). Beyond T2D status, efforts have been made to identify genetic markers of treatment response, specifically related to action or pharmacology of traditional diabetes treatments [78, 79, 46, 80-83]. Unfortunately, to date, these efforts have failed to reveal gene variants relating specifically to pharmacology or treatment response to diabetes medicines that meaningfully inform genetically-guided therapeutic strategies. Similarly, while modest efforts have been made to prospectively define treatment response according to simple clinical features or according to detailed physiologic measures, these have not been successful overall [84-86].

\section{Areas for future research}

One obvious need is to scale up projects like TOPMed, IUGC PHI, and UK Biobank, which are the next wave of ambitious projects in T2D genomics and precision health. For these efforts to 
become a reality, the scale that has been observed in GWAS studies will be needed. Additionally, many of these omics are influenced by environment and timing of measurement in the disease course. Along these lines is the need for better measures of environment and also measurement at multiple times across the lifespan.

One opportunity for understanding the interplay of environment on genetics is afforded by populations with distinct patterns of environmental exposure, which has been discussed in a detailed review [87]. WGS and thorough temporal assessments of exposures and outcomes conducted in ever larger biobanks and health care settings will greatly advance our understanding of interactions, causal exposures and mechanisms [87].

The most common form of diabetes in children and adolescents historically has been type 1 diabetes (T1D), but this is no longer the case as youth are increasingly being diagnosed with T2D. In the USA, findings from the SEARCH for Diabetes in Youth study showed a substantial increase in the prevalence of T2D from 2001 to 2009 [88], with the incidence of T2D in Asian and Pacific Islander youth and in African American youth now greater than that of T1D [89, 90]. Over $90 \%$ of young patients report a family history of diabetes, which suggests a significant genetic contribution to the incidence in youth [90]. The role of genetic factors contributing to the development of T2D in youth is further supported by epidemiological evidence observing rates are most common in high diabetes prevalence racial groups [91]. At the same time, the unabated increase in childhood obesity is also tightly linked to the emergence of T2D as a new type of pediatric diabetes [92]. Early results from the RISE study show the clinical course is markedly different in adolescents compared to adults suggesting the need for further research to better prevent and manage this unique and increasingly important population [93-96].

\section{Conclusions}

The main concept of 'precision health' in T2D is to move away from generalized treatment approaches to prevention and treatment decisions that are personalized and based on individual variability in genes, environment, and lifestyle. The achievements accomplished by genomics holds promise for identifying novel mechanisms and classifying T2D into homogeneous clinical subtypes. In the new era in which technological developments coupled with expanded computational power and increased statistical sophistication, a number of major initiatives are underway with the objective of creating big-data environments for the global query of discrete biological axes. Efforts are also being made in translating the enormous quantity of information into useful health knowledge and applying this to define the most appropriate therapeutic strategies for both disease prevention and management. Meanwhile, before precision diabetes becomes common clinical and public health practice, there is much to resolve, for instance, how to improve the reproducibility of results from big data, and what is the best multidisciplinary approach to leverage big data in precision health.

\section{Compliance with Ethical Standards}

Conflict of Interest Jennifer Wessel and Yuan Lin declare that they have no conflict of interest.

Human and Animal Rights and Informed Consent This article does not contain any studies with human or animal subjects performed by any of the authors. 


\section{References}

Papers of particular interest, published recently, have been highlighted as:

- Of importance

1. Shaw JE, Sicree RA, Zimmet PZ. Global estimates of the prevalence of diabetes for 2010 and 2030. Diabetes research and clinical practice. 2010;87(1):4-14.

doi:10.1016/j.diabres.2009.10.007.

2. Tuomi T, Santoro N, Caprio S, Cai M, Weng J, Groop L. The many faces of diabetes: a disease with increasing heterogeneity. Lancet (London, England). 2014;383(9922):1084-94. doi:10.1016/s0140-6736(13)62219-9.

3. Zimmet PZ, Magliano DJ, Herman WH, Shaw JE. Diabetes: a 21st century challenge. Lancet Diabetes Endocrinol. 2014;2(1):56-64. doi:10.1016/S2213-8587(13)70112-8.

4. McCarthy MI. Painting a new picture of personalised medicine for diabetes. Diabetologia. 2017;60(5):793-9. doi:10.1007/s00125-017-4210-x.

5. McPherson JD, Marra M, Hillier L, Waterston RH, Chinwalla A, Wallis $\mathrm{J}$ et al. A physical map of the human genome. Nature. 2001;409(6822):934-41. doi:10.1038/35057157.

6. International HapMap Consortium. The International HapMap Project. Nature. 2003;426(6968):789-96. doi:10.1038/nature02168.

7. Mahajan A, Taliun D, Thurner M, Robertson NR, Torres JM, Rayner NW et al. Fine-mapping type 2 diabetes loci to single-variant resolution using high-density imputation and islet-specific epigenome maps. Nature genetics. 2018;50(11):1505-13. doi:10.1038/s41588-018-0241-6. 8. Mahajan A, Wessel J, Willems SM, Zhao W, Robertson NR, Chu AY et al. Refining the accuracy of validated target identification through coding variant fine-mapping in type 2 diabetes. Nature genetics. 2018;50(4):559-71. doi:10.1038/s41588-018-0084-1.

9. Gaulton KJ, Ferreira T, Lee Y, Raimondo A, Magi R, Reschen ME et al. Genetic fine mapping and genomic annotation defines causal mechanisms at type 2 diabetes susceptibility loci. Nature genetics. 2015;47(12):1415-25. doi:10.1038/ng.3437.

10. Merino J, Florez JC. Precision medicine in diabetes: an opportunity for clinical translation. Ann N Y Acad Sci. 2018;1411(1):140-52. doi:10.1111/nyas.13588.

11. Manolio TA, Collins FS, Cox NJ, Goldstein DB, Hindorff LA, Hunter DJ et al. Finding the missing heritability of complex diseases. Nature. 2009;461(7265):747-53.

doi:10.1038/nature08494.

12. Fuchsberger C, Flannick J, Teslovich TM, Mahajan A, Agarwala V, Gaulton KJ et al. The genetic architecture of type 2 diabetes. Nature. 2016;536(7614):41-7. doi:10.1038/nature18642. 13. Consortium STD, Estrada K, Aukrust I, Bjorkhaug L, Burtt NP, Mercader JM et al. Association of a low-frequency variant in HNF1A with type 2 diabetes in a Latino population. Jama. 2014;311(22):2305-14. doi:10.1001/jama.2014.6511.

14. Moltke I, Grarup N, Jorgensen ME, Bjerregaard P, Treebak JT, Fumagalli M et al. A common Greenlandic TBC1D4 variant confers muscle insulin resistance and type 2 diabetes. Nature. 2014;512(7513):190-3. doi:10.1038/nature13425.

15. Floyd JS, Psaty BM. The Application of Genomics in Diabetes: Barriers to Discovery and Implementation. Diabetes care. 2016;39(11):1858-69. doi:10.2337/dc16-0738.

16. Khera AV, Chaffin M, Aragam KG, Haas ME, Roselli C, Choi SH et al. Genome-wide polygenic scores for common diseases identify individuals with risk equivalent to monogenic mutations. Nature genetics. 2018;50(9):1219-24. doi:10.1038/s41588-018-0183-z. 
17. Pallares-Mendez R, Aguilar-Salinas CA, Cruz-Bautista I, Del Bosque-Plata L. Metabolomics in diabetes, a review. Ann Med. 2016;48(1-2):89-102. doi:10.3109/07853890.2015.1137630.

18. Piening BD, Zhou W, Contrepois K, Rost H, Gu Urban GJ, Mishra T et al. Integrative Personal Omics Profiles during Periods of Weight Gain and Loss. Cell Syst. 2018;6(2):157-70 e8. doi:10.1016/j.cels.2017.12.013.

19. Rhee EP, Yang Q, Yu B, Liu X, Cheng S, Deik A et al. An exome array study of the plasma metabolome. Nat Commun. 2016;7:12360. doi:10.1038/ncomms12360.

20. O'Connell TM. The complex role of branched chain amino acids in diabetes and cancer. Metabolites. 2013;3(4):931-45. doi:10.3390/metabo3040931.

21. Wang TJ, Ngo D, Psychogios N, Dejam A, Larson MG, Vasan RS et al. 2-Aminoadipic acid is a biomarker for diabetes risk. J Clin Invest. 2013;123(10):4309-17. doi:10.1172/JCI64801.

22. Lopez-Villar E, Martos-Moreno GA, Chowen JA, Okada S, Kopchick JJ, Argente J. A proteomic approach to obesity and type 2 diabetes. J Cell Mol Med. 2015;19(7):1455-70. doi:10.1111/jcmm.12600.

23. O'Connell TM, Markunas CA. DNA Methylation and MicroRNA-Based Biomarkers for Risk of Type 2 Diabetes. Curr Diabetes Rev. 2016;12(1):20-9.

24. Hartstra AV, Bouter KE, Backhed F, Nieuwdorp M. Insights into the role of the microbiome in obesity and type 2 diabetes. Diabetes care. 2015;38(1):159-65. doi:10.2337/dc14-0769.

25. Grarup N, Sandholt CH, Hansen T, Pedersen O. Genetic susceptibility to type 2 diabetes and obesity: from genome-wide association studies to rare variants and beyond. Diabetologia. 2014;57(8):1528-41. doi:10.1007/s00125-014-3270-4.

26. Pleis JR, Lucas JW, Ward BW. Summary health statistics for U.S. adults: National Health Interview Survey, 2008. Vital and health statistics Series 10, Data from the National Health Survey. 2009(242):1-157.

27. Hannon TS, Bacha F, Lin Y, Arslanian SA. Hyperinsulinemia in African-American adolescents compared with their American white peers despite similar insulin sensitivity: a reflection of upregulated beta-cell function? Diabetes care. 2008;31(7):1445-7.

doi: $10.2337 / \mathrm{dc0} 8-0116$.

28. Vistisen D, Witte DR, Tabak AG, Herder C, Brunner EJ, Kivimaki M et al. Patterns of obesity development before the diagnosis of type 2 diabetes: the Whitehall II cohort study. PLoS Med. 2014;11(2):e1001602. doi:10.1371/journal.pmed.1001602.

29. Unwin N, Shaw J, Zimmet P, Alberti KG. Impaired glucose tolerance and impaired fasting glycaemia: the current status on definition and intervention. Diabet Med. 2002;19(9):708-23. 30. Choi KM, Lee J, Kim DR, Kim SK, Shin DH, Kim NH et al. Comparison of ADA and WHO criteria for the diagnosis of diabetes in elderly Koreans. Diabet Med. 2002;19(10):853-7. 31. Sadikot SM, Nigam A, Das S, Bajaj S, Zargar AH, Prasannakumar KM et al. Comparing the ADA 1997 and the WHO 1999 criteria: Prevalence of Diabetes in India Study. Diabetes research and clinical practice. 2004;66(3):309-15. doi:10.1016/j.diabres.2004.04.009. 32. Botas P, Delgado E, Castano G, Diaz de Grenu C, Prieto J, Diaz-Cadorniga FJ. Comparison of the diagnostic criteria for diabetes mellitus, WHO-1985, ADA-1997 and WHO-1999 in the adult population of Asturias (Spain). Diabet Med. 2003;20(11):904-8.

33. Boj SF, van Es JH, Huch M, Li VS, Jose A, Hatzis $P$ et al. Diabetes risk gene and Wnt effector Tcf7I2/TCF4 controls hepatic response to perinatal and adult metabolic demand. Cell. 2012;151(7):1595-607. doi:10.1016/j.cell.2012.10.053.

34. Nicolson TJ, Bellomo EA, Wijesekara N, Loder MK, Baldwin JM, Gyulkhandanyan AV et al. Insulin storage and glucose homeostasis in mice null for the granule zinc transporter ZnT8 and studies of the type 2 diabetes-associated variants. Diabetes. 2009;58(9):2070-83.

doi:10.2337/db09-0551.

35. Kido Y, Burks DJ, Withers D, Bruning JC, Kahn CR, White MF et al. Tissue-specific insulin resistance in mice with mutations in the insulin receptor, IRS-1, and IRS-2. J Clin Invest.

2000;105(2):199-205. doi:10.1172/JCI7917. 
36. Hashimoto N, Kido Y, Uchida T, Asahara S, Shigeyama Y, Matsuda T et al. Ablation of PDK1 in pancreatic beta cells induces diabetes as a result of loss of beta cell mass. Nature genetics. 2006;38(5):589-93. doi:10.1038/ng1774.

37. Lyssenko V, Eliasson L, Kotova O, Pilgaard K, Wierup N, Salehi A et al. Pleiotropic effects of GIP on islet function involve osteopontin. Diabetes. 2011;60(9):2424-33. doi:10.2337/db101532.

38. Mussig K, Staiger H, Machicao F, Haring HU, Fritsche A. Genetic variants affecting incretin sensitivity and incretin secretion. Diabetologia. 2010;53(11):2289-97. doi:10.1007/s00125-0101876-8.

39. Andersson SA, Olsson AH, Esguerra JL, Heimann E, Ladenvall C, Edlund A et al. Reduced insulin secretion correlates with decreased expression of exocytotic genes in pancreatic islets from patients with type 2 diabetes. Mol Cell Endocrinol. 2012;364(1-2):36-45.

doi:10.1016/j.mce.2012.08.009.

40. Gutierrez-Aguilar R, Kim DH, Casimir M, Dai XQ, Pfluger PT, Park J et al. The role of the transcription factor ETV5 in insulin exocytosis. Diabetologia. 2014;57(2):383-91.

doi:10.1007/s00125-013-3096-5.

41. Dimas AS, Lagou V, Barker A, Knowles JW, Magi R, Hivert MF et al. Impact of type 2 diabetes susceptibility variants on quantitative glycemic traits reveals mechanistic heterogeneity. Diabetes. 2014;63(6):2158-71. doi:10.2337/db13-0949.

42. Florez JC, Jablonski KA, Bayley N, Pollin TI, de Bakker PI, Shuldiner AR et al. TCF7L2 polymorphisms and progression to diabetes in the Diabetes Prevention Program. The New England journal of medicine. 2006;355(3):241-50. doi:10.1056/NEJMoa062418.

43. Delahanty LM, Pan Q, Jablonski KA, Watson KE, McCaffery JM, Shuldiner A et al. Genetic predictors of weight loss and weight regain after intensive lifestyle modification, metformin treatment, or standard care in the Diabetes Prevention Program. Diabetes care. 2012;35(2):363-6. doi:10.2337/dc11-1328.

44. Pollin TI, Jablonski KA, McAteer JB, Saxena R, Kathiresan S, Kahn SE et al. Triglyceride response to an intensive lifestyle intervention is enhanced in carriers of the GCKR Pro446Leu polymorphism. J Clin Endocrinol Metab. 2011;96(7):E1142-7. doi:10.1210/jc.2010-2324.

45. Hivert MF, Jablonski KA, Perreault L, Saxena R, McAteer JB, Franks PW et al. Updated genetic score based on 34 confirmed type 2 diabetes Loci is associated with diabetes incidence and regression to normoglycemia in the diabetes prevention program. Diabetes.

2011;60(4):1340-8. doi:10.2337/db10-1119.

46. Zhou K, Donnelly L, Yang J, Li M, Deshmukh H, Van Zuydam N et al. Heritability of variation in glycaemic response to metformin: a genome-wide complex trait analysis. Lancet Diabetes Endocrinol. 2014;2(6):481-7. doi:10.1016/S2213-8587(14)70050-6.

47. Gatza ML, Silva GO, Parker JS, Fan C, Perou CM. An integrated genomics approach identifies drivers of proliferation in luminal-subtype human breast cancer. Nature genetics. 2014;46(10):1051-9. doi:10.1038/ng.3073.

48. Li L, Cheng WY, Glicksberg BS, Gottesman O, Tamler R, Chen R et al. Identification of type 2 diabetes subgroups through topological analysis of patient similarity. Sci Transl Med.

2015;7(311):311ra174. doi:10.1126/scitransImed.aaa9364.

49. Wessel J, Chu AY. Integrative Genomic Approach Identifies Molecular Subtypes of Type 2 Diabetes. Diabetes.65(Supplement 1). doi:https://doi.org/10.2337/db16-1-381.

50. Ahlqvist E, Storm P, Karajamaki A, Martinell M, Dorkhan M, Carlsson A et al. Novel subgroups of adult-onset diabetes and their association with outcomes: a data-driven cluster analysis of six variables. Lancet Diabetes Endocrinol. 2018;6(5):361-9. doi:10.1016/S22138587(18)30051-2.

51. Udler MS, Kim J, von Grotthuss M, Bonas-Guarch S, Cole JB, Chiou J et al. Type 2 diabetes genetic loci informed by multi-trait associations point to disease mechanisms and 
subtypes: A soft clustering analysis. PLoS Med. 2018;15(9):e1002654.

doi:10.1371/journal.pmed.1002654.

52. Reitman ML, Schadt EE. Pharmacogenetics of metformin response: a step in the path toward personalized medicine. J Clin Invest. 2007;117(5):1226-9. doi:10.1172/JCI32133. 53. Schadt EE, Lamb J, Yang X, Zhu J, Edwards S, Guhathakurta D et al. An integrative genomics approach to infer causal associations between gene expression and disease. Nature genetics. 2005;37(7):710-7. doi:10.1038/ng1589.

54. Ritchie MD, Holzinger ER, Li R, Pendergrass SA, Kim D. Methods of integrating data to uncover genotype-phenotype interactions. Nat Rev Genet. 2015;16(2):85-97.

doi:10.1038/nrg3868.

55. Multhaup ML, Seldin MM, Jaffe AE, Lei X, Kirchner H, Mondal P et al. Mouse-human experimental epigenetic analysis unmasks dietary targets and genetic liability for diabetic phenotypes. Cell Metab. 2015;21(1):138-49. doi:10.1016/j.cmet.2014.12.014.

56. Cancer Genome Atlas Network. Comprehensive molecular portraits of human breast tumours. Nature. 2012;490(7418):61-70. doi:10.1038/nature11412.

57. Cancer Genome Atlas Research Network, Brat DJ, Verhaak RG, Aldape KD, Yung WK, Salama SR et al. Comprehensive, Integrative Genomic Analysis of Diffuse Lower-Grade Gliomas. The New England journal of medicine. 2015;372(26):2481-98.

doi:10.1056/NEJMoa1402121.

58. Encode Project Consortium. An integrated encyclopedia of DNA elements in the human genome. Nature. 2012;489(7414):57-74. doi:10.1038/nature11247.

59. GTEx Consortium. Human genomics. The Genotype-Tissue Expression (GTEx) pilot analysis: multitissue gene regulation in humans. Science. 2015;348(6235):648-60.

doi:10.1126/science.1262110.

60. Mele M, Ferreira PG, Reverter F, DeLuca DS, Monlong J, Sammeth M et al. Human genomics. The human transcriptome across tissues and individuals. Science. 2015;348(6235):660-5. doi:10.1126/science.aaa0355.

61. Rivas MA, Pirinen M, Conrad DF, Lek M, Tsang EK, Karczewski KJ et al. Human genomics. Effect of predicted protein-truncating genetic variants on the human transcriptome. Science. 2015;348(6235):666-9. doi:10.1126/science.1261877.

62. Gaulton KJ, Nammo T, Pasquali L, Simon JM, Giresi PG, Fogarty MP et al. A map of open chromatin in human pancreatic islets. Nature genetics. 2010;42(3):255-9. doi:10.1038/ng.530. 63. Pasquali L, Gaulton KJ, Rodriguez-Segui SA, Mularoni L, Miguel-Escalada I, Akerman I et al. Pancreatic islet enhancer clusters enriched in type 2 diabetes risk-associated variants. Nature genetics. 2014;46(2):136-43. doi:10.1038/ng.2870.

64. Varshney A, Scott LJ, Welch RP, Erdos MR, Chines PS, Narisu N et al. Genetic regulatory signatures underlying islet gene expression and type 2 diabetes. Proc Natl Acad Sci U S A. 2017;114(9):2301-6. doi:10.1073/pnas.1621192114.

65. Type 2 Diabetes Knowledge Portal. www.type2diabetesgenetics.org/. Accessed October 2018.

66. Fitipaldi H, McCarthy MI, Florez JC, Franks PW. A Global Overview of Precision Medicine in Type 2 Diabetes. Diabetes. 2018;67(10):1911-22. doi:10.2337/dbi17-0045.

67. Collins FS, Varmus H. A new initiative on precision medicine. The New England journal of medicine. 2015;372(9):793-5. doi:10.1056/NEJMp1500523.

68. Indiana University Grand Challenge. https://grandchallenges.iu.edu/precision-

health/index.html. Accessed November 2018.

69. Trans-Omics for Precision Medicine (TOPMed) Program.

https://www.nhlbi.nih.gov/science/trans-omics-precision-medicine-topmed-program. Accessed November 2018. 
70. Brody JA, Morrison AC, Bis JC, O'Connell JR, Brown MR, Huffman JE et al. Analysis commons, a team approach to discovery in a big-data environment for genetic epidemiology. Nature genetics. 2017;49(11):1560-3. doi:10.1038/ng.3968.

71. Knowler WC, Barrett-Connor E, Fowler SE, Hamman RF, Lachin JM, Walker EA et al. Reduction in the incidence of type 2 diabetes with lifestyle intervention or metformin. The New England journal of medicine. 2002;346(6):393-403. doi:10.1056/NEJMoa012512.

72. Pan XR, Li GW, Hu YH, Wang JX, Yang WY, An ZX et al. Effects of diet and exercise in preventing NIDDM in people with impaired glucose tolerance. The Da Qing IGT and Diabetes Study. Diabetes care. 1997;20(4):537-44.

73. Tuomilehto J, Lindstrom J, Eriksson JG, Valle TT, Hamalainen H, Ilanne-Parikka P et al Prevention of type 2 diabetes mellitus by changes in lifestyle among subjects with impaired glucose tolerance. The New England journal of medicine. 2001;344(18):1343-50. doi:10.1056/NEJM200105033441801.

74. Hivert MF, Christophi CA, Franks PW, Jablonski KA, Ehrmann DA, Kahn SE et al. Lifestyle and Metformin Ameliorate Insulin Sensitivity Independently of the Genetic Burden of Established Insulin Resistance Variants in Diabetes Prevention Program Participants. Diabetes. 2016;65(2):520-6. doi:10.2337/db15-0950.

75. Wessel J, Marrero DG. Genetic Testing for Type 2 Diabetes in High-Risk Children: the Case for Primordial Prevention. Research Ideas and Outcomes. 2017;3(e20695).

76. Zeevi D, Korem T, Zmora N, Israeli D, Rothschild D, Weinberger A et al. Personalized Nutrition by Prediction of Glycemic Responses. Cell. 2015;163(5):1079-94. doi:10.1016/j.cell.2015.11.001.

77. Mutie PM, Giordano GN, Franks PW. Lifestyle precision medicine: the next generation in type 2 diabetes prevention? BMC Med. 2017;15(1):171. doi:10.1186/s12916-017-0938-X. 78. Anderson SL, Trujillo JM, McDermott M, Saseen JJ. Determining predictors of response to exenatide in type 2 diabetes. J Am Pharm Assoc (2003). 2012;52(4):466-71. doi:10.1331/JAPhA.2012.10217.

79. Florez JC, Jablonski KA, Taylor A, Mather K, Horton E, White NH et al. The C allele of ATM rs11212617 does not associate with metformin response in the Diabetes Prevention Program. Diabetes care. 2012;35(9):1864-7. doi:10.2337/dc11-2301.

80. Florez JC. The pharmacogenetics of metformin. Diabetologia. 2017;60(9):1648-55. doi:10.1007/s00125-017-4335-y.

81. GoDARTS and UKPDS Diabetes Pharmacogenetics Study Group, Wellcome Trust Case Control Consortium, Zhou K, Bellenguez C, Spencer CC, Bennett AJ et al. Common variants near ATM are associated with glycemic response to metformin in type 2 diabetes. Nature genetics. 2011;43(2):117-20. doi:10.1038/ng.735.

82. Zhou K, Yee SW, Seiser EL, van Leeuwen N, Tavendale R, Bennett AJ et al. Variation in the glucose transporter gene SLC2A2 is associated with glycemic response to metformin. Nature genetics. 2016;48(9):1055-9. doi:10.1038/ng.3632.

83. Feng Y, Mao G, Ren X, Xing H, Tang G, Li Q et al. Ser1369Ala variant in sulfonylurea receptor gene ABCC8 is associated with antidiabetic efficacy of gliclazide in Chinese type 2 diabetic patients. Diabetes care. 2008;31(10):1939-44. doi:10.2337/dc07-2248.

84. Fu H, Cao D, Boye KS, Curtis B, Schuster DL, Kendall DM et al. Early Glycemic Response Predicts Achievement of Subsequent Treatment Targets in the Treatment of Type 2 Diabetes: A Post hoc Analysis. Diabetes Ther. 2015;6(3):317-28. doi:10.1007/s13300-015-0119-x. 85. Martono DP, Lub R, Lambers Heerspink HJ, Hak E, Wilffert B, Denig P. Predictors of response in initial users of metformin and sulphonylurea derivatives: a systematic review. Diabet Med. 2015;32(7):853-64. doi:10.1111/dme.12688.

86. Shah M, Varghese RT, Miles JM, Piccinini F, Dalla Man C, Cobelli C et al. TCF7L2 Genotype and alpha-Cell Function in Humans Without Diabetes. Diabetes. 2016;65(2):371-80. doi:10.2337/db15-1233. 
87. Franks PW, McCarthy MI. Exposing the exposures responsible for type 2 diabetes and obesity. Science. 2016;354(6308):69-73. doi:10.1126/science.aaf5094.

88. Dabelea D, Bell RA, D'Agostino RB, Jr., Imperatore G, Johansen JM, Linder B et al. Incidence of diabetes in youth in the United States. Jama. 2007;297(24):2716-24.

doi:10.1001/jama.297.24.2716.

89. Liu LL, Yi JP, Beyer J, Mayer-Davis EJ, Dolan LM, Dabelea DM et al. Type 1 and Type 2 diabetes in Asian and Pacific Islander U.S. youth: the SEARCH for Diabetes in Youth Study. Diabetes care. 2009;32 Suppl 2:S133-40. doi:10.2337/dc09-S205.

90. Mayer-Davis EJ, Beyer J, Bell RA, Dabelea D, D'Agostino R, Jr., Imperatore G et al. Diabetes in African American youth: prevalence, incidence, and clinical characteristics: the SEARCH for Diabetes in Youth Study. Diabetes care. 2009;32 Suppl 2:S112-22. doi:10.2337/dc09-S203.

91. Gill-Carey O, Hattersley AT. Genetics and type 2 diabetes in youth. Pediatr Diabetes. 2007;8 Suppl 9:42-7. doi:10.1111/j.1399-5448.2007.00331.x.

92. Hannon TS, Rao G, Arslanian SA. Childhood obesity and type 2 diabetes mellitus. Pediatrics. 2005;116(2):473-80. doi:10.1542/peds.2004-2536.

93. Buse JB, D'Alessio DA, Riddle MC. Can We RISE to the Challenge of Youth-Onset Type 2 Diabetes? Diabetes care. 2018;41(8):1560-2. doi:10.2337/dci18-0025.

94. Rise Consortium. Metabolic Contrasts Between Youth and Adults With Impaired Glucose Tolerance or Recently Diagnosed Type 2 Diabetes: II. Observations Using the Oral Glucose Tolerance Test. Diabetes care. 2018;41(8):1707-16. doi:10.2337/dc18-0243.

95. Rise Consortium. Metabolic Contrasts Between Youth and Adults With Impaired Glucose Tolerance or Recently Diagnosed Type 2 Diabetes: I. Observations Using the Hyperglycemic Clamp. Diabetes care. 2018;41(8):1696-706. doi:10.2337/dc18-0244.

96. Rise Consortium. Impact of Insulin and Metformin Versus Metformin Alone on beta-Cell Function in Youth With Impaired Glucose Tolerance or Recently Diagnosed Type 2 Diabetes. Diabetes care. 2018;41(8):1717-25. doi:10.2337/dc18-0787.

Fig. 1 Precision health of diabetes: the molecular and epidemiologic heterogeneity of T2D and the need for re-defining T2D into mechanistic and clinical subtypes. An overview of our current understanding of T2D and how future research can transform T2D into mechanistically similar subtypes for improved prevention and management. 
Expanded Assessment of Environment Electronic health records

Mobile digital technologies

Prospective epidemiologic studies

Environmental data

Social determinants

Multi-omic measures

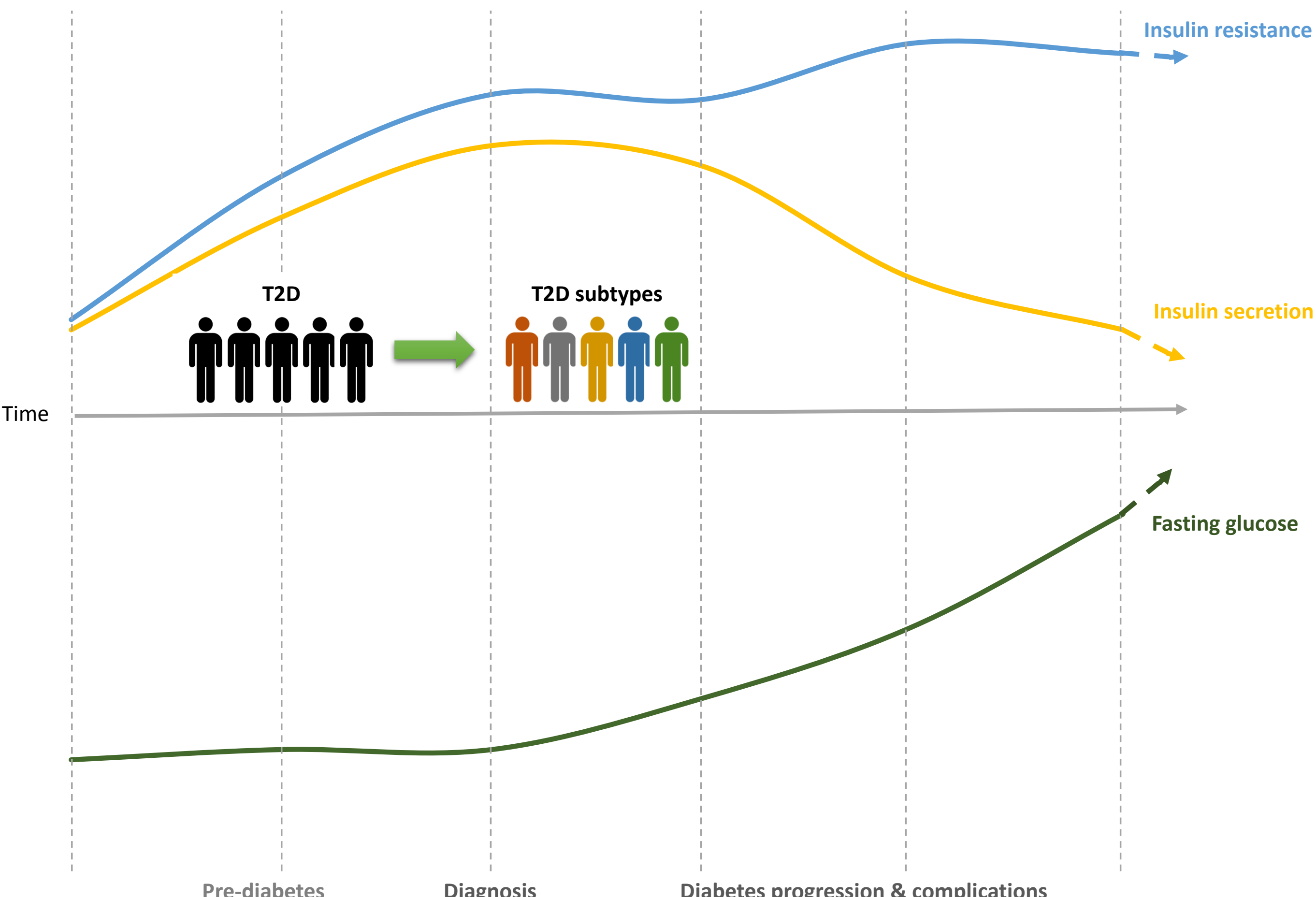

Expanded Multi-Omic Data

Large-scale genomic annotations of diabetes-specific tissue

Large-scale genotyping or whole genome sequencing

Large-scale multi-omic measures 